McGill/92-30

hep-th/9205086

May 92

\title{
Fractional Superspace Formulation of Generalized Super-Virasoro Algebras
}

\author{
StÉPhANe DuranD* \\ Department of Physics \\ McGill University \\ Ernest Rutherford Building \\ 3600 University Street \\ Montréal, PQ, Canada H3A 2T8
}

\begin{abstract}
We present a fractional superspace formulation of the centerless parasuper-Virasoro and fractional super-Virasoro algebras. These are two different generalizations of the ordinary super-Virasoro algebra generated by the infinitesimal diffeomorphisms of the superline. We work on the fractional superline parametrized by $t$ and $\theta$, with $t$ a real coordinate and $\theta$ a paragrassmann variable of order $M$ and canonical dimension $1 / F$. We further describe a more general structure labelled by $M$ and $F$ with $M \geq F$. The case $F=2$ corresponds to the parasuper-Virasoro algebra of order $M$, while the case $F=M$ leads to the fractional super-Virasoro algebra of order $F$. The ordinary super-Virasoro algebra is recovered at $F=M=2$. The connection with $q$-oscillator algebras is discussed.
\end{abstract}

* E-mail address: durand@hep.physics.mcgill.ca 
Symmetries play a fundamental role in physics. Therefore, all new kinds of symmetry are worth studying. For instance, supersymmetries could be a key ingredient of unifying theories. Up to now, at least two generalizations of supersymmetry are known: the para-supersymmetries ${ }^{[1,2]}$ which already possess interesting applications in quantum mechanics ${ }^{[1,3,4]}$, and the fractional supersymmetries ${ }^{[5,6,7]}$ which appear, in particular, in the context of the chiral Potts model ${ }^{[8]}$. In this letter, we shall present a new algebraic stucture that allows the unification of the concepts of para-superalgebra and fractional superalgebra, and that furthermore describes new types of symmetries. More precisely, we present a novel generalization of the centerless super-Virasoro algebra which we will call a generalized super-Virasoro algebra. This construction has the following features:

- It is parametrized by 2 positive integers $M$ and $F$ with $M \geq F$.

- It possesses a $Z_{F}$ grading.

- It describes operators of fractional spin $s=1+\frac{1}{F}$.

- It is realized within the framework of a fractional superspace formalism using paragrassmann variables of order $M$ and canonical dimension $1 / F$.

- It contains as particular cases, the parasuper-Virasoro algebras of order $M$ (for $F=2$ ) and the fractional super-Virasoro algebras of order $F$ (for $M=F$ ). The ordinary super-Virasoro algebra is recovered at $F=M=2$.

Thus, it also provides a new unified realization of the "old" fractional and para super-Virasoro algebras. (In Ref. [2] the latter was realized in terms of the Green representation ${ }^{[9]}$ while in Refs. $[5,6,7]$ the former was strictly realized in matrix form).

Let us first recall some standard results. The well-known centerless superVirasoro algebra is given by

$$
\begin{aligned}
& {\left[L_{m}, L_{n}\right]=(m-n) L_{m+n}} \\
& {\left[L_{n}, G_{r}\right]=\left(\frac{1}{2} n-r\right) G_{n+r}} \\
& \left\{G_{r}, G_{s}\right\}=2 L_{r+s}
\end{aligned}
$$

with $m, n \in Z$ and $r, s \in Z+\frac{1}{2}$. When centrally extended, it yields the NeveuSchwarz algebra which plays a central role in superstring theories. The algebraic relations (1), referred to as the two-dimensional superconformal algebra, can also be thought of as realized by the generators of the infinitesimal diffeomorphisms 
$\left(L_{n}\right)$ and superdiffeomorphisms $\left(G_{r}\right)$ of the superline:

$$
\begin{aligned}
& L_{n}=t^{1-n} \partial_{t}-\frac{1}{2}(n-1) t^{-n} \theta \partial_{\theta}, \quad n \in Z \\
& G_{r}=t^{\frac{1}{2}-r}\left(\partial_{\theta}+\theta \partial_{t}\right), \quad r \in Z+\frac{1}{2}
\end{aligned}
$$

where $t$ is the real line parameter $\left(\partial_{t} \equiv \partial / \partial t\right)$ and $\theta$ a paragrassmann variable satifying $\left(\partial_{\theta} \equiv \partial / \partial \theta\right)$ :

$$
\theta^{2}=0, \quad\left\{\partial_{\theta}, \theta\right\}=1
$$

The generators $L_{n}$ and $G_{r}$ respectively have spin 2 and $3 / 2$. Generally speaking, one says that $\phi_{n}$ has conformal spin $s$ if

$$
\left[L_{m}, \phi_{n}\right]=((s-1) m-n) \phi_{m+n}
$$

Note that $L_{1}, L_{0}, L_{-1}, G_{1 / 2}$ and $G_{-1 / 2}$ span an $O S p(1,1)$ superalgebra and that $L_{1}$ and $G_{1 / 2}$ alone also form a superalgebra. We will come back to these subalgebras in the context of quantum mechanics. Note also that the algebra (1) might have been presented with $(1 c)$ replaced by

$$
G_{r}^{2}=L_{2 r}
$$

since $(1 c)$ can be reconstructed ${ }^{a}$ from (5) using $(1 a, b)$. In the following, the commutation relation ( $1 a)$ will stay unchanged, $(1 b)$ will keep the same form but (1c) will be completely modified, being replaced by a multilinear product.

We shall use the fractional superspace formalism introduced in Ref. [10]. Very recently, a detailed analysis of this formalism with a generalization to many paragrassmann variables appeared in Ref. [11]. We shall work on a space parametrized by a real coordinate $t$, a paragrassmann variable $\theta$ of order $M$, and a prime root of unity $q \in C$, satisfying

$$
\theta^{M}=0, \quad q^{M}=1 \quad\left(q^{n} \neq 1 \text { for } n<M\right) \quad M=1,2, \ldots .
$$

By a prime root, we mean a root satisfying the condition in parenthesis, i.e., for a given order, we keep only roots which differ from those of all lower orders $(e . g$., $q \neq \pm 1$ for $M=4$ ). We also need another parameter $F$ (called the fractional order) defined by

$$
\operatorname{dim}\left(\theta^{F}\right)=\operatorname{dim}(t), \quad F=1,2, \ldots
$$


Later, we will choose $M \geq F$. The derivative $\partial_{\theta} \equiv \partial / \partial \theta$, which also satisfies $\partial_{\theta}^{M}=0$, is introduced in the following way

$$
\partial_{\theta} \cdot \theta=1+q \theta \partial_{\theta}
$$

We recover the ordinary grassmann case (3) for $M=2$ and the bosonic one for $M \rightarrow \infty$. The definition (8) implies

$$
\partial_{\theta} \cdot \theta^{n}=\frac{1-q^{n}}{1-q} \theta^{n-1}+q^{n} \theta^{n} \partial_{\theta}
$$

with

$$
\frac{1-q^{n}}{1-q}=1+q+q^{2}+\ldots+q^{n-1} \quad(=0 \text { for } n=M) .
$$

A matrix realization of $\theta$ and $\partial_{\theta}$ is given in Ref. [11]. Note that equation (8), which can be rewritten in the form

$$
\left[\partial_{\theta}, \theta\right]_{q} \equiv \partial_{\theta} \theta-q \theta \partial_{\theta}=1
$$

corresponds to one of the defining relations of the $q$-oscillator algebra ${ }^{[12]}$. We will return to this connection later.

We now introduce the generalized generators $L_{n}^{(F ; M)}$ and $G_{r}^{(F ; M)}$ of the infinitesimal diffeomorphisms of the fractional superline. They are of fractional order $F$ and paragrassmann order $M$ with $M \geq F$ :

$$
\begin{gathered}
L_{n}^{(F ; M)}=t^{1-n} \partial_{t}-\frac{1}{F}(n-1) t^{-n} B^{(M)}, \quad n \in Z \\
G_{r}^{(F ; M)}=t^{\frac{1}{F}-r}\left(\partial_{\theta}+e \theta^{F-1} \partial_{t}\right)-e\left(r-\frac{1}{F}\right) t^{\frac{1}{F}-r-1} \theta^{F-1} B^{(M)}, \quad r \in Z+\frac{1}{F}
\end{gathered}
$$

with

$$
B^{(M)}=\sum_{i=0}^{M-1} c_{i} \theta^{i} \partial_{\theta}{ }^{i}=c_{0}+\sum_{i=1}^{M-1} c_{i} \theta^{i} \partial_{\theta}{ }^{i}
$$

and where

$$
c_{0}: \text { arbitrary, } \quad c_{i}=\frac{(1-q)^{i}}{1-q^{i}}, \quad i=1,2, \ldots, M-1 .
$$

Now, $\theta$ and $\partial_{\theta}$ satisfy relations $(6,7,8)$. The constant $e$ is not fixed for the moment. The generators (2) of the supersymmetric case are recovered for $F=M=2$ 
and $c_{0}=0$. The operators (12) satisfy the following generalized super-Virasoro algebras:

$$
\begin{gathered}
{\left[L_{m}, L_{n}\right]=(m-n) L_{m+n}} \\
{\left[L_{n}, G_{r}\right]=\left(\frac{1}{F} n-r\right) G_{n+r}} \\
G_{r}^{M}=\sum_{i=1}^{\llbracket M / F \rrbracket}(-)^{i+1} a_{i} G_{r}^{M-F i} L_{F r}^{i}
\end{gathered}
$$

with $L_{n} \equiv L_{n}^{(F ; M)}, G_{r} \equiv G_{r}^{(F ; M)}$, and where $\llbracket M / F \rrbracket$ is the integer part of $M / F$. The coefficients $a_{i}$ are given by

$$
a_{i} \equiv a_{i}^{(F ; M)}=\frac{M}{i}\left[\frac{e}{(1-q)^{F-1}}\right]^{i} b_{i}^{(F ; M)}
$$

where

$$
b_{i}^{(F ; M)}=\frac{(M-i F+i-1) !}{(i-1) !(M-i F) !} .
$$

[The factor $\left(e /(1-q)^{F-1}\right)^{i}$ in the coefficients $a_{i}$ may be absorbed in the definition of the $\left.L_{n}.\right]$ From $(4)$ and $(15 b)$, we see that $G_{r}^{(F ; M)}$ has fractional spin $1+1 / F$. Note that $a_{1}^{(F ; M)}=e M /(1-q)^{F-1}$. From now on, we set $e=(1-q)^{F-1} / M$ so that $a_{1}^{(F ; M)}=1$.

The operator $B^{(M)}$ given in (13) is such that, for any order $M$, it satisfies:

$$
\left[B^{(M)}, \theta\right]=\theta, \quad\left[B^{(M)}, \partial_{\theta}\right]=-\partial_{\theta}
$$

This makes contact with quantum algebras since eqs. (11) and (17) are actually a particular case (for $i=j=1$ and $b_{1}=\partial_{\theta}, b_{1}^{\dagger}=\theta$ ) of the defining relations of multi $q$-oscillator algebra ${ }^{[12]}$ :

$$
\left[b_{i}, b_{i}^{\dagger}\right]_{q}=1, \quad\left[b_{i}, b_{j}^{\dagger}\right]=0 \quad(i \neq j), \quad\left[N, b_{i}\right]=-b_{i}, \quad\left[N, b_{i}^{\dagger}\right]=b_{i}^{\dagger} .
$$

The formula (17) allows one to compute exactly the commutation relation (15b). However, (15c) has been verified only for particular $F$ and $M$.

Let us point out some limiting cases of formulas (15c). First, for $F=M$ we recover the fractional super-Virasoro algebras of order $F$ :

$$
G_{r}^{F}=L_{F r}
$$


The trivial case $F=1$ shows that $G_{r}^{(1 ; 1)}=e L_{r}^{(1 ; 1)}$. At $F=2$, we recover the supersymmetric case (5) which is equivalent to $(1 c)$ as already mentionned. Actually, this kind of equivalence exists for all orders $F$. Indeed, from $(15 a, b)$ and (19), one may derive ${ }^{b}$ the general formula

$$
\left\{G_{r_{1}}, G_{r_{2}}, \ldots, G_{r_{F}}\right\}=F ! L_{r_{1}+r_{2}+\ldots+r_{F}}
$$

where we have used the following definition of the multilinear symmetric product

$$
\left\{X_{1}, X_{2}, \ldots, X_{M}\right\} \equiv X_{1} X_{2} \ldots X_{M}+\text { all permutations of the } X_{i} \text {. }
$$

(For instance, for $M=3$ the right hand side of (21) contains 6 terms.) This symmetric product is a kind of generalization of the anti-commutator and $(1 c)$ is a particular case of (20). Eqs. $(15 a, b)$ and (20) define the centerless fractional super-Virasoro algebras ${ }^{[5,6,7]}$ which have a $Z_{F}$ grading. (In Ref. [7], these algebras are presented in an interesting different form.) Finally, these structures possess a subalgebra generated by $Q \equiv G_{1 / F}=\left(\partial_{\theta}+e \theta^{F-1} \partial_{t}\right)$ and $H \equiv L_{1}=\partial_{t}$ :

$$
Q^{F}=H, \quad[H, Q]=0
$$

Thus $Q$ appears as a fractional covariant derivative. For $F>2$, this is the only subalgebra (actually, we may add $L_{0}$ ). This subalgebra has arisen in the context of the chiral Potts model ${ }^{[8]}$.

The second limiting case consists of the parasuper-Virasoro algebras of order $M$ which are recovered at $F=2$. Here, it is more difficult to present these algebras in a simpler form than in $(15 c)$; therefore we shall make them explicit for a few cases (we have set $a_{i}^{(2 ; M)} \equiv a_{i}^{(M)}$ ):

$$
\begin{array}{ll}
M=2: & G_{r}^{2}=L_{2 r} \\
M=3: & G_{r}^{3}=G_{r} L_{2 r} \\
M=4: & G_{r}^{4}=G_{r}^{2} L_{2 r}-a_{2}^{(4)} L_{2 r}^{2} \\
M=5: & G_{r}^{5}=G_{r}^{3} L_{2 r}-a_{2}^{(5)} G_{r} L_{2 r}^{2} \\
M=6: & G_{r}^{6}=G_{r}^{4} L_{2 r}-a_{2}^{(6)} G_{r}^{2} L_{2 r}^{2}+a_{3}^{(6)} L_{2 r}^{3} \\
\text { etc. } &
\end{array}
$$


As before, we can reconstruct ${ }^{c}$ the multilinear product from $(15 a, b)$ and $(23)$. For instance, we find

$$
\begin{array}{ll}
M=3: & \left\{G_{r}, G_{s}, G_{t}\right\}=G_{r} L_{s+t}+\text { perm. } \\
M=4: \quad & \left\{G_{r}, G_{s}, G_{t}, G_{u}\right\}=G_{r} G_{s} L_{t+u}+a_{2}^{(4)} L_{r+s} L_{t+u}+\text { perm. }
\end{array}
$$

Now "perm." signifies that one should add the terms which are obtained from those already appearing in the right-hand side of (24) by performing all permutations of $(r, s, t, \ldots)$. The structure relations $(15 a),(15 b)$ for $F=2$, and $(24)$ define the centerless parasuper-Virasoro algebras which have a $Z_{2}$ grading. (The coefficients $a_{i}^{(M)}$ are not the same as in Ref. [2].) The smallest subalgebra is generated as before by $Q \equiv G_{1 / 2}=\left(\partial_{\theta}+e \theta \partial_{t}\right)$ and $H \equiv L_{1}=\partial_{t}$. From (15b) and (23) we find $[H, Q]=0$ and $Q^{2}=H$ for $M=2, Q^{3} \cong Q H$ for $M=3, Q^{4} \cong Q^{2} H-H^{2}$ for $M=4$, etc, which are the defining relations of the parasupersymmetric quantum mechanics of order $M^{[4]}$, where $Q$ is the parasupercharge and $H$ the hamiltonian. Actually, there exists a larger subalgebra given by $L_{1}, L_{0}, L_{-1}, G_{1 / 2}$ and $G_{-1 / 2}$ (this is not true for $F>2$ ) which spans the paragrassmann generalization of the $\operatorname{OSp}(1,1)$ superalgebra. In this regard, notice that the parasuperconformal quantum mechanics of order $M=3$ has been constructed in Ref. [3].

All the other cases $(M \neq F \neq 2)$ are new symmetry algebras. For instance, the intermediate case $F=3$ and $M=6$ reduces for $r=1 / 3$ to

$$
Q^{6}=a_{1} Q^{3} H-a_{2} H^{2}
$$

with the $a_{i}$ given by $(16 a)$. It is also possible to generate a multi-index version of (15c) for all of these novel cases.

Before concluding, let us write an interesting formula. For a given $q$ of order $M$, one has

$$
\sum_{\left(n_{1}, \ldots, n_{i}\right) \in S_{i}} \prod_{j=1}^{i}\left(1-q^{n_{j}}\right)=\left(\begin{array}{c}
M \\
i
\end{array}\right) \equiv \frac{M !}{i !(M-i) !}
$$

for $S_{i}$ the set of combinations of $i$ numbers out of the set $\{1,2, \ldots, M-1\}$. As particular cases, one finds for $i=1$ and $i=M-1$ :

$$
\sum_{j=1}^{M-1}\left(1-q^{j}\right)=\prod_{j=1}^{M-1}\left(1-q^{j}\right)=M
$$


The next natural step is to compute the central extension of these generalized super-Virasoro algebras. Maybe, one would find central terms with a dependence on $F, M$ and $D$, leading to new critical dimensions $D$ for $M \neq 2$.

\section{Acknowledgments}

I am pleased to thank Luc Vinet and Vyacheslav Spiridonov for their interest and useful discussions. This work is supported in part through funds provided by the Natural Sciences and Engineering Research Council (NSERC) of Canada and the Fonds FCAR of the Québec Ministry of Education.

\section{Notes}

(a) More explicitly, (1c) is reconstructed from $\left[L_{r-s}, G_{s}^{2}\right]=\left[L_{r-s}, L_{2 s}\right]$. Strictly speaking, we should also add $\left\{G_{s}, G_{3 s}\right\}=2 L_{4 s}$ to (5). However, this equation follows if we assume that the (anti-)commutation relations have a regular structure.

(b) For instance, for $F=3$ we must successively commute each side of the equation (19) with $L_{s-r}$ and $L_{t-r}$. Strictly speaking, we should here add $\left\{G_{r}, G_{4 r}, G_{4 r}\right\}=6 L_{9 r}$ to (19). This equation follows if we assume a regular structure.

(c) Now, for $M=3$ for instance, we must perform the same trick on (23) as the one mentioned in the preceding note but adding the relation $(24 a)$ with $s=3 r$ and $t=3 r$. 


\section{References}

[1] V.A. Rubakov and V.P. Spiridonov, Mod. Phys. Lett. A3, 1337 (1988).

[2] S. Durand, R. Floreanini, M. Mayrand and L. Vinet, Phys. Lett. B233, 158 (1989).

[3] S. Durand and L. Vinet, Mod. Phys. Lett. A4, 2519 (1989); S. Durand and L. Vinet, in Field Theory and Particle Physics, edited by O. J. P. Eboli, M. Gomes and A. Santoro (World Scientific, Singapore, 1990) pp. 291-314. For a review, see Ref. [6].

[4] S. Durand, M. Mayrand, V.P. Spiridonov and L. Vinet, Mod. Phys. Lett. A6, 3163 (1991).

[5] S. Durand, Parasupersymétrie, Ph.D. thesis, University of Montréal (1990).

[6] S. Durand, R. Floreanini, M. Mayrand, V.P. Spiridonov and L. Vinet, "Parasupersymmetries and Fractional Supersymmetries", in Quantum Field Theory, Quantum Mechanics and Quantum Optics, Proceedings of the XVIIIth International Colloquium on Group Theoretical Methods in Physics, 4-9 June 1990, Moscow, USSR, edited by V.V. Dodonov and V.I. Man'ko (Nova Science Publishers, 1991) pp. 145-154.

[7] T. Nakanishi, Progr. Theor. Phys. 82, 207 (1989); Mod. Phys. Lett. A3, 1507 (1988).

[8] D. Bernard and V. Pasquier, Saclay preprint SPhT/89-284 (1989); D. Bernard and A. Leclair, Saclay preprint SPhT/90-082 (1990).

[9] Y. Ohnuki and S. Kamefuchi, Quantum Field Theory and Parastatistics, (Springer-Verlag, New York, 1982).

[10] C. Ahn, D. Bernard and A. Leclair, Nucl. Phys. B346, 409 (1990).

[11] A.T. Filippov, A.P. Isaev and A.B. Kurdikov, "Paragrassmann Analysis and Quantum Groups", JINR Dubna preprint (April 1992) hep-th/9204089.

[12] See for instance: R. Floreanini and L. Vinet, Lett. Math. Phys. 22, 45 (1991); "Representations of Quantum Algebras and q-Special Functions" in IIth International Wigner Symposium, edited by V. Dobrev and H. Dobner (SpringerVerlag, Berlin, 1992); V.P. Spiridonov, private communication. 